\title{
METHODS OF MEASURING THE INDUCTANCES OF LOW-RESISTANCE STANDARDS
}

\author{
By Frank Wenner, Ernest Weibel, and F. B. Silsbee
}

\section{INTRODUCTION}

\section{GENERAL USE OF LOW-RESISTANCE STANDARDS}

In precise measurements of alternating-current phenomena it frequently becomes necessary to use low-resistance standards of large current capacity. The most common example is in the calibration of instruments for the accurate measurement of power in alternating-current circuits. Power is usually measured by a wattmeter in connection with current and voltage transformers, and these transformers are in turn calibrated by the use of standards of known resistance and inductance. An error in the phase angle of a standard will appear in the apparent phase angle of the transformer, and hence enter directly into the power measurement. For example, if a $0.000 \mathrm{r}-\mathrm{ohm}$ standard is assumed to be noninductive when its inductance is $10 \mathrm{~cm}$, or $10 \times 10^{-9}$ henry, the current in the standard at 60 cycles will lag $2.15^{\circ}$ behind the electromotive force at its potential terminals, and the error in measuring power at a power factor of $0.7 \mathrm{I}$ would be 3.7 percent.

\section{DEFINITION OF RESISTANCE AND INDUCTANCE}

The low-resistance standards considered in this paper are conductors having two pairs of terminals, namely, current and potential terminals. We shall treat only such conductors in which when a current

$$
i=I_{m} \sin p t
$$

flows through the current terminals, the difference of potential between the potential terminals is 


$$
e=A \sin p t+B \cos p t
$$

The resistance $R$ is $A / I_{m}$, the inductance $L$ is $B / p I_{m}$, the time constant $T$ is $B / p A=L / R$, and the phase angle $\theta$ is $\tan ^{-1} B / A=$ $\tan ^{-1} p L / R$ where $p=2 \pi$ times the frequency.

In order that the resistance and inductance of a low-resistance standard be definite, ${ }^{1}$ it is necessary to have the potential and

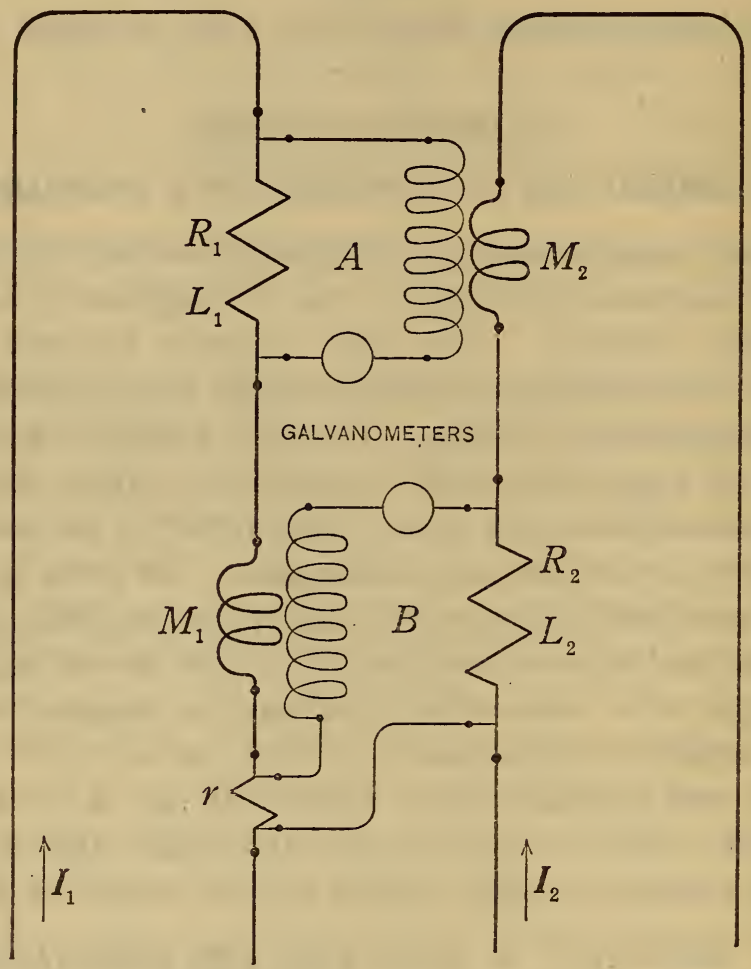

FIG. I.-Simple diagram of connections for measuring sum of phase angles of two resistance standards

current leads brought out in a definite manner. In a welldesigned standard for general use in alternating-current measurements the resistance and inductance are practically independent of the maniner of making the connections to the terminals of the standard. 


\section{PREVIOUS MEASUREMENTS}

All values heretofore obtained for the residual inductance of "noninductive" standards of low resistance have been based upon the calculated inductance of some conductor. Such a calculation can only be performed for simple shapes and for ideal conditions as regards current distribution, current and potential leads, end effects, etc., so that the construction of a standard of which the inductance can be calculated with sufficient certainty offers some difficulties.

The inductances of standards of odd shapes have then been obtained by measuring the differences between their phase angles and that of a calculated standard. These difference measurements have been made, using a Rosa curve tracer, ${ }^{2}$ an electrometer, ${ }^{3}$ an electrodynamometer, ${ }^{4}$ a Thomson bridge, ${ }^{5}$ and a current transformer. ${ }^{6}$

The two methods described in this paper for determining the phase angles of low-resistance standards without recourse to computed values are believed to be the only ones now available.

\section{THEORY OF METHODS}

\section{FIRST METHOD}

This method gives primarily the sum of the phase angles of two resistance standards. By using three resistance standards three sums $^{7}$ can be measured from which each phase angle can be obtained.

The resistance standards are each connected in series with a mutual inductance, as indicated in Fig. I, and excited by alternating currents substantially in quadrature. The constants of the circuits and the relative phase and magnitude of the two currents are then adjusted so that no current flows in either galvanometer.

\footnotetext{
2 By plotting curves of the voltages across the potential terminals of 2 resistance standards carrying the same current, Dr. Rosa obtained directly the difference of the phase angles.

${ }^{3}$ Orlich, Zs. für Instk., 29, p. 24I; 1909.

${ }^{4}$ Agnew and Fitch, this Bulletin, 6, p. 28r; r909; Scientific Paper No. I30.

${ }^{5}$ Sharp \& Crawford, Trans. Amer. Inst. E. E., 29, p. 1540; 19ro. Wenner, this Bulletin, 8, p. 603; I9r2.

${ }^{6}$ Agnew and Silsbee, Trans. A. I. E. E., 31, p. I635; 1912.

${ }^{7}$ By measuring the sum of the phase angles of two resistance standards by this method and the difference by any of those mentioned above, one can determine both phase angles.
}

$6844^{\circ}-15-2$ 
If then we let the real parts of $\boldsymbol{I}_{1} \varepsilon^{j p t}$ and $\boldsymbol{I}_{2} \varepsilon^{j p t}$ (where $\boldsymbol{I}_{1}$ and $\boldsymbol{I}_{2}$ are complex quantities or vectors and $j$ represents the $\sqrt{-I}$ ) represent the instantaneous values of the currents, and $R_{1}, L_{1}, M_{1}, r$, $R_{2}, L_{2}$, and $M_{2}$ represent the constants of the circuits, we have

and

$$
\left(R_{1}+j p L_{1}\right) I_{1}=j p M_{2} I_{2}
$$

$$
\left(R_{2}+j p L_{2}\right) \boldsymbol{I}_{2}=\left(r-j p M_{1}\right) \boldsymbol{I}_{1}
$$

Eliminating $\boldsymbol{I}_{1} / \boldsymbol{I}_{2}$, we get

or

$$
\frac{j p M_{2}}{R_{1}+j p L_{1}}=\frac{R_{2}+j p L_{2}}{r-j p M_{1}}
$$

$$
j r p M_{2}+p^{2} M_{1} M_{2}=R_{1} R_{2}+j\left(p L_{1} R_{2}+p L_{2} R_{1}\right)-p^{2} L_{1} L_{2} .
$$

Therefore

and

$$
R_{1} R_{2}=p^{2}\left(M_{1} M_{2}+L_{1} L_{2}\right)
$$

$$
\frac{p L_{1}}{R_{1}}+\frac{p L_{2}}{R_{2}}=\frac{r p M_{2}}{R_{1} R_{2}} \text { or } \frac{r}{p M_{1}}
$$

very closely. The first of these equations, (6), can be used in the absolute measurement of resistance and the second gives a measure of the sum of the phase angles of the two resistances.

The above relations can be shown very simply by the vector diagram shown in Fig. 2. If $Z_{1}$ and $Z_{2}$ are the impedances of the two resistances, then $Z_{1} I_{1}$ leads $I_{1}$ by $\theta_{1}=\tan ^{-1} p L_{1} / R_{1}$, and when the galvanometer $A$ carries no current this equals $-j p M_{2} I_{2}$, so that $I_{2}$ must lead $Z_{1} I_{1}$ by $90^{\circ}$. Now, $Z_{2} I_{2}$ leads $I_{2}$ by $\theta_{2}=\tan ^{-1}$ $p L_{2} / R_{2}$, as shown. Also $-j p\left(-M_{1}\right) I_{1}$ or $j p M_{1} I_{1}$ leads $I_{1}$ by $90^{\circ}$, as shown. From this we subtract $r I_{1}$ parallel to $I_{1}$; and when the galvanometer $\mathrm{B}$ carries no current we know that $j p M_{1} \boldsymbol{I}_{1}-r \boldsymbol{I}_{1}=$ $Z_{2} I_{2}$. By inspection of the diagram we see that

$$
\tan \left(\theta_{1}+\theta_{2}\right)=\frac{r}{p M_{1}}
$$

and if $\theta_{1}$ and $\theta_{2}$ are small we have

$$
\frac{p L_{1}}{R_{1}}+\frac{p L_{2}}{R_{2}}=\frac{r}{p M_{1}}
$$

very closely. A modification of this method of measuring the sum is to use, instead of the small resistance $r$, a small mutual 
inductance $m$, whose primary is in series with $R_{2}$ and whose secondary is in series with galvanometer $B$. The sum of the phase angles would then be $p m / R_{2}$ instead of $r / p M_{1}$.

In the above proofs it is assumed that the mutual inductances are pure; i. e., give electromotive forces in exact quadrature with the primary current. Any deviation from quadrature represents an equal error in the measurement of the sum of the phase angles.

It is also assumed that the currents are sinusoidal. If this is not the case, there will be unbalanced harmonic electromotive forces in the galvanometer circuits. The detectors must therefore

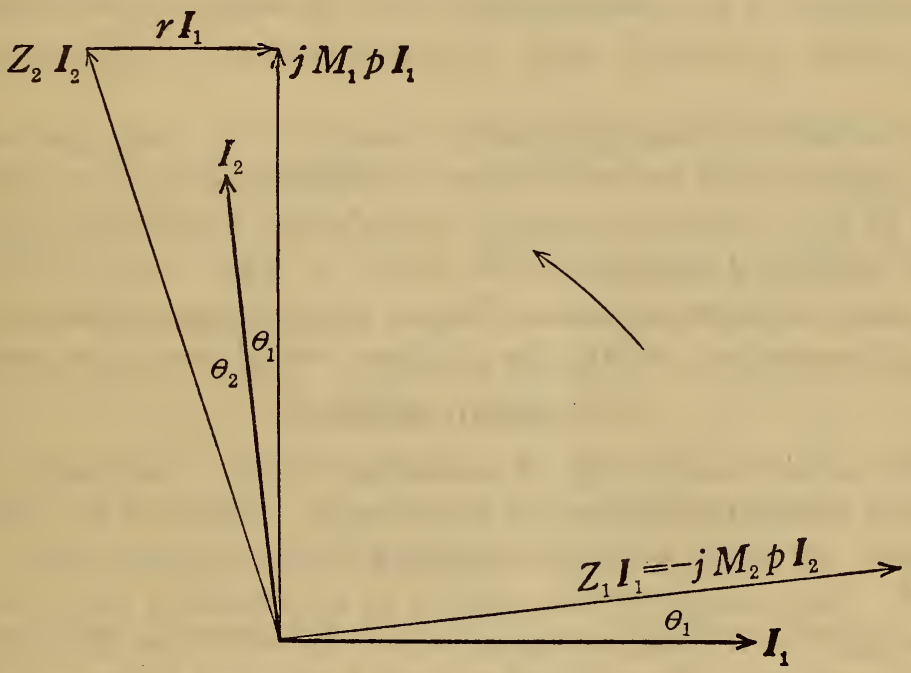

FIG. 2.-Vector diagram of electromotive forces in measurement of sum of phase angles of two resistance standards

be sufficiently selective so that these electromotive forces give only negligible deflections. A tuned vibration galvanometer is very selective. If it is not sufficiently so, it is possible by using an electrical resonating system in series with it to increase greatly the selectiveness of the arrangement. The use of such a system, however, necessarily introduces resistance into the galvanometer circuit, and thus reduces somewhat the sensitivity.

The sensitivity of the arrangement is easily calculated, since we know that for a lack of balance in phase of one minute there is $0.0003 R_{1} I_{1}$ volt in circuit $\mathrm{A}$ and $0.0003 R_{2} I_{2}$ volt in circuit $\mathrm{B}$. 
The deflections produced by these voltages can be calculated from the constants of the galvanometers and galvanometer circuits.

In order to obtain as large a voltage as possible for a given difference in phase, the mutual inductances should be of such magnitudes and of such construction as will allow both resistances to be used at full current capacity. The resistances of the secondaries of the mutual inductances should be kept low in order not to diminish unduly the deflection produced by this voltage.

Since the balance depends directly upon the frequency, it is necessary that the speed of the generators be kept very constant. For example, if the measurement is to be made to one minute of phase angle, the speed must be kept constant to about 0.03 per. cent.

In making the measurement one observer who reads galvanometer A controls the ratio and phase difference of the two currents so as to keep his galvanometer undeflected, while the other observer reading galvanometer $\mathrm{B}$ varies $M_{1}$ and $r$ (or $m$ ) so as to bring the deflection to zero. With a simultaneous balance of the two galvanometer circuits the relations stated above are satisfied.

\section{SECOND METHOD}

This method makes use of a standard whose resistance can be changed without changing its inductance. This can be done, for example, by using a copper standard whose temperature can be varied. The measurement consists in determining the change in phase angle or in time constant when the resistance is changed a known amount. Then, if the observed decrease in phase angle is $\Delta \theta$, or in time constant is $\Delta T$, and the corresponding proportional increase in resistance be $a$, we have

and

$$
\frac{p L}{R}=\frac{\mathrm{r}+a}{a} \Delta \theta
$$

$$
\frac{L}{R}=\frac{\mathrm{I}+a}{a} \Delta T
$$

very closely. To show this, we make use of the known relations (See Fig. 3.)

$$
\tan \theta=\frac{p L}{R} \text { and } \tan (\theta-\Delta \theta)=\frac{p L}{(\mathrm{I}+a) R}
$$


Since the angles are small, these give

$$
\Delta \theta=\frac{p L}{R}-\frac{p L}{(\mathrm{I}+a) R}=\frac{p L}{R} \frac{a}{\mathrm{I}+a}
$$

This method requires that there be no change in form or in current distribution sufficient to cause an appreciable change in inductance. Systematic errors due to this cause can be eliminated by the use of well-constructed standards of different designs.

The change in phase angle can be measured by any of the methods used for measuring differences of phase angles referred to on page $\mathrm{I} 3$. The precision attainable by the use of this method

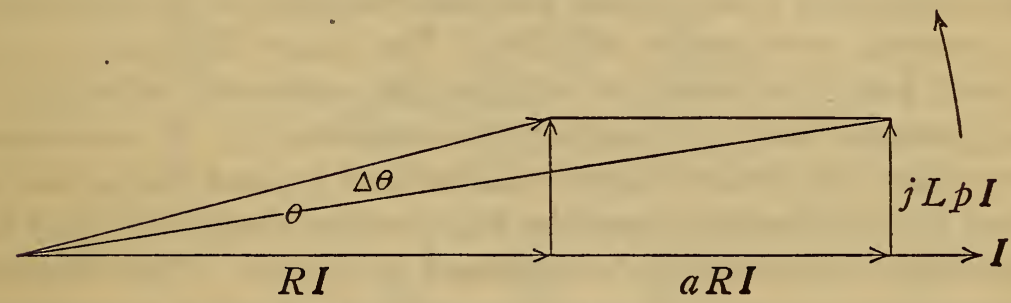

FIG. 3.-Vector diagram showing change in phase angle with change in resistance

for measuring the phase angle of a standard is determined by the precision of measurement of the change in phase angle and by the magnitude of the proportional change in resistance. Thus, if $a=0.5$, which with a copper resistance corresponds to a change from 0 to $I I 7^{\circ} \mathrm{C}$, and it is desired to measure $p L / R$ to I minute, it is necessary to measure $\Delta \theta$ to $\frac{0.5}{\mathrm{I}+0.5}$, or about 0.3 minute.

\section{EXPERIMENTAL WORK}

\section{STANDARDS}

In order to test the practicability of these methods, measurements have been made on four low resistances. These were all made of flat-strip resistance material, and the current and potential leads were brought out in a definite manner. The values of the resistances and the current capacities in oil are given in Table I. $R$ and $S$ were of manganin, $K$ of kulmitz metal, and $C$ of copper. The standards $C$ and $K$ were such that the inductance could be calculated from the dimensions with some certainty. 


\section{FIRST METHOD}

All of the six possible sums of the phase angles of the four standards were measured by the first method. To do this, the apparatus was connected as shown in Fig. 4. $R_{1}$ and $R_{2}$ are the resistances to be measured; $r_{1}, r_{2}$, and $r_{3}$ are resistances for obtaining the adjustable low resistance $r$ in Fig. I. For example, with $r_{1}=0.00 \mathrm{I} \mathrm{ohm}, r_{2}=0.1 \mathrm{ohm}$, and $r_{3}=\mathrm{IOO} \mathrm{ohms}, r=\mathrm{IO}^{-6} \mathrm{ohm} . \quad r_{3}$ was variable so that $r$ could be easily varied over a considerable range. The two circuits were excited through transformers from two generators directly connected to a motor. By manipulation of the resistance $r_{5}$ and $r_{6}$ and the field rheostats of the generators the currents were easily adjusted. The phase difference of the currents could be varied by rotating the stationary armature of one generator and by changing the resistance $r_{7}$. It was necessary to use the double transformation and ground connection as shown to avoid leakage from the high-voltage circuits through the measuring circuits and galvanometers to ground. The vibration galvanometers were of the moving coil type having a resistance of about $\mathrm{I} .5 \mathrm{ohm}$, a voltage sensitivity of $0.16 \mathrm{~mm}$ per microvolt, and an amplitude time constant of 2 seconds. The frequency was 60 cycles per second. A resonating system consisting of an inductance of 0.14 henry in series with a capacity of 50 microfarads was used in series with each galvanometer. These were found to be very effective. The impedance at the fundamental frequency was about I ohm, while to the third harmonic it was about I 40 ohms.

A Maxwell bridge with a charge and discharge commutator on the shaft of the generators was used to indicate changes in speed. The motor was run from a storage battery, so that the speed was very constant.

The procedure followed in making the measurements was practically as described above. In addition reversals of current and potential connections of the resistances and reversals of the primary and secondary connections of the mutual inductances were made in various combinations in order to eliminate errors due to stray alternating magnetic fields not considered in the theory above. 


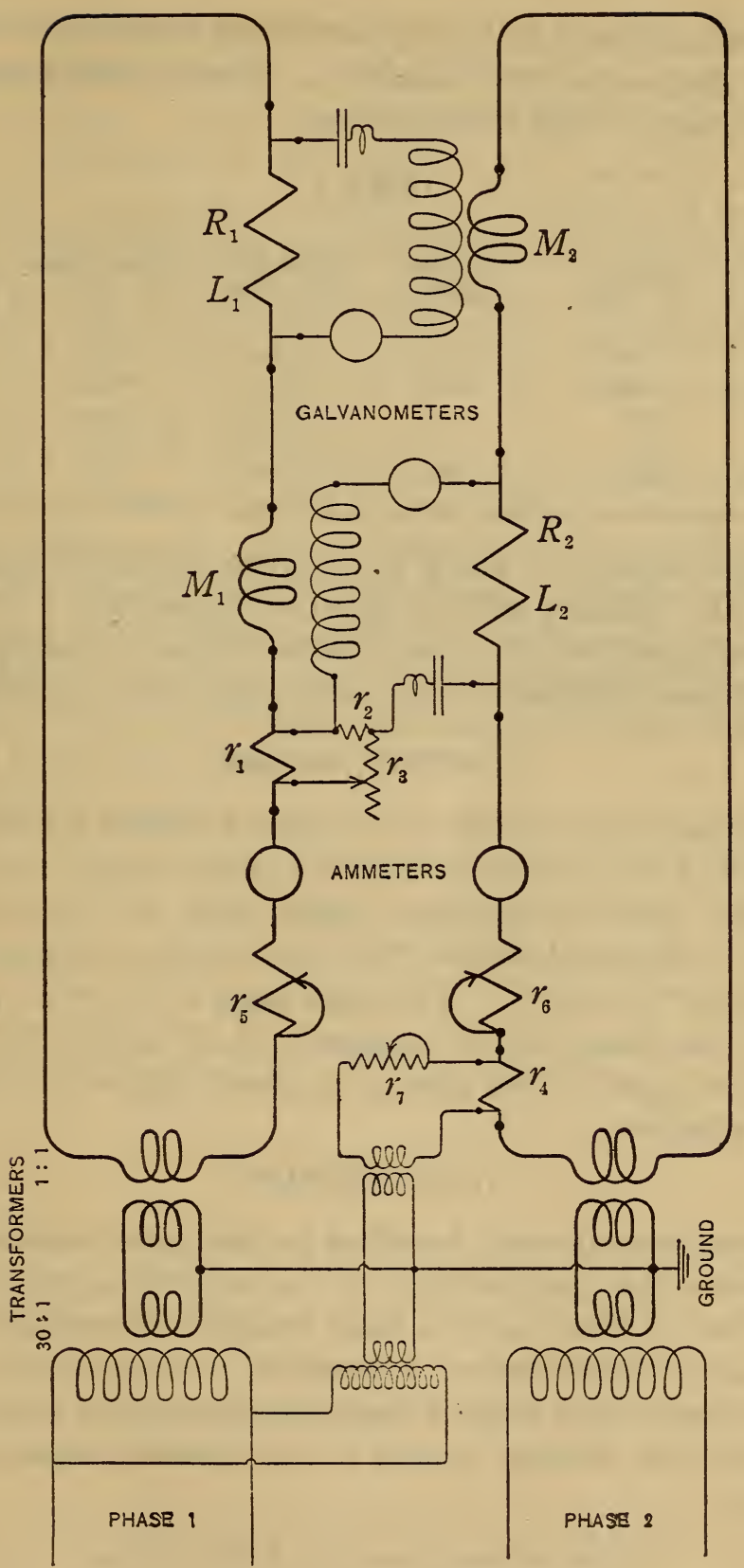

FIG. 4.-Diagram of connections as used in measuring sum of phase sangle of two resistance standards 
The phase angles at 60 cycles determined from the observations on the six sums are given in Table $\mathrm{r}$. These values satisfy all of the observations within two minutes.

TABLE 1

\begin{tabular}{c|c|c|c|c|c}
\hline Designation & Resistance & $\begin{array}{c}\text { Current } \\
\text { capacity }\end{array}$ & $\begin{array}{c}\text { Phase angle at } \\
60 \text { cycles }\end{array}$ & $\begin{array}{c}\text { Time constant } \\
\times 10^{6}\end{array}$ & Inductance \\
\cline { 2 - 5 } & ohm & amp. & min. & sec. & cm. \\
C & 0.000337 & 300 & 29.0 & 22.4 & 7.6 \\
K & 0.01 & 75 & 2.5 & 1.9 & 19.0 \\
R & 0.001 & 200 & 19.0 & 14.7 & 14.7 \\
S & 0.001 & 500 & 16.5 & 12.7 & 12.7 \\
\hline
\end{tabular}

The phase angles of $C$ and $K$ at 60 cycles calculated from dimensions are 24.I minutes and 0.8 minutes, respectively. The values determined experimentally are therefore higher, showing that the ideal conditions assumed in the calculations were not fulfilled.

\section{SECOND METHOD}

Measurements were made by the second method on standard $C$. The standard was connected in the primary circuit of a current transformer and the apparent phase angle of the transformer measured by the usual method, ${ }^{8}$ with the shunt at various temperatures from $20^{\circ} \mathrm{C}$ to $80^{\circ} \mathrm{C}$. The phase angle at 60 cycles computed, assuming the change in the apparent phase angle of the transformer to be equal to the change in phase angle of the standard, was 30.5 minutes.

\section{CONCLUSIONS}

The experimental work described in this paper was undertaken to determine the practicability of the methods suggested above, with a view to their use in a more complete investigation of the inductance of four-terminal conductors by one of the authors. It served also to show some of the precautions which must be taken in case the first method is used in the absolute measurement of resistance. 
The first method is rather complicated and requires much special apparatus, but affords a valuable check on results obtained by other methods. In the work described here the precision was limited by fluctuations in the speed of the generators, but it is believed that the precision attained can be improved by further attention to this and other limiting factors. As has been pointed out above (p. I 5), the results depend directly upon the magnitude and purity of the mutual inductances used, and these are to be investigated further in connection with the work on the inductance of four-terminal conductors.

The second method was found to be much simpler, and with the current-transformer method of measuring the change in phase angle and resistance is easily carried out. A precision of o.I minute in the measurement of the change in phase angle, at 60 cycles, should be attainable; so with an increase of roo per cent in the resistance the phase angle could be measured to $0.2 \mathrm{~min}$ ute. The accuracy would probably be slightly lower than this on account of systematic errors.

Washington, August 5, I9I4. 


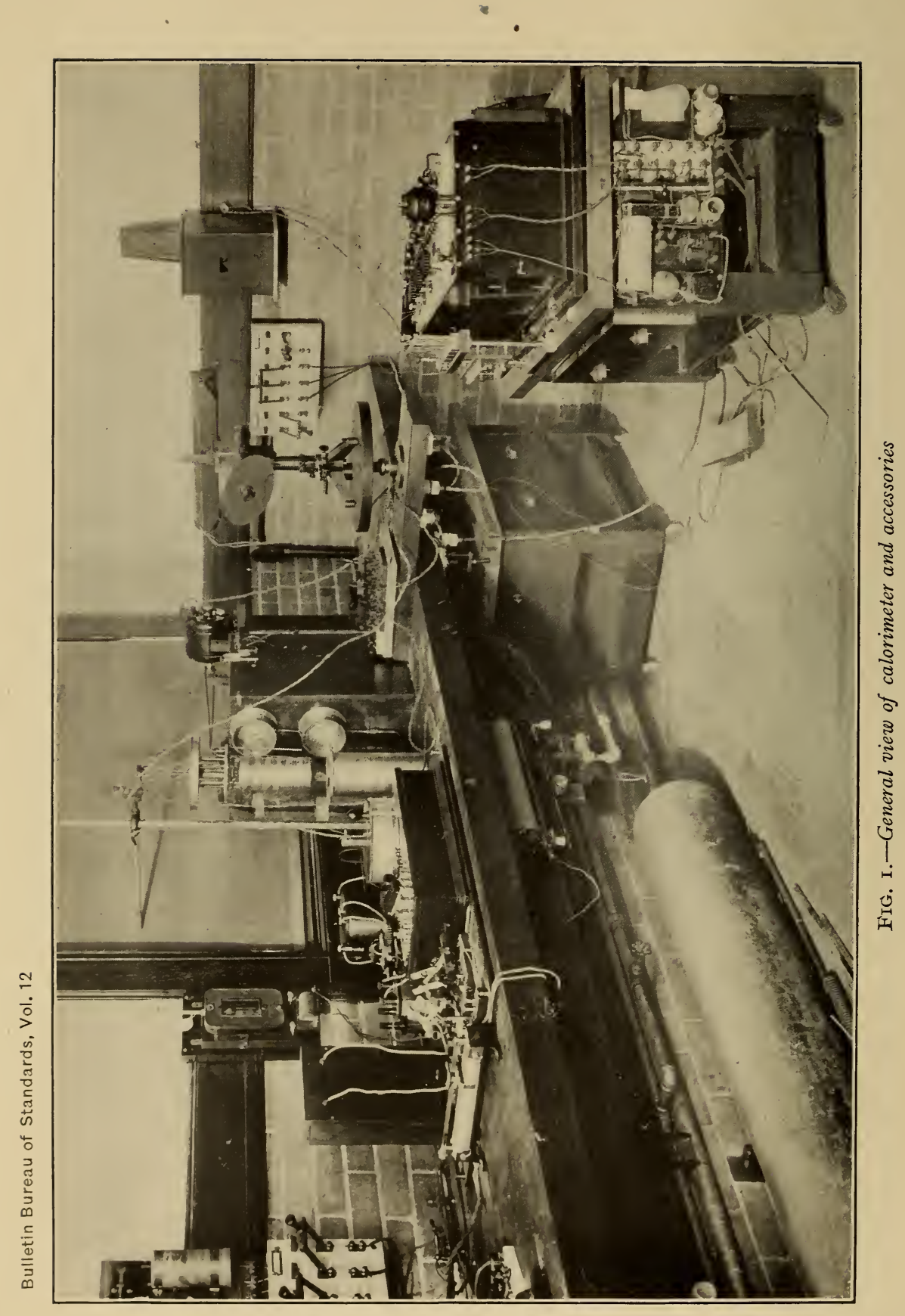

